livraisons

d'Histoire

de l'Architecture

\section{Livraisons de l'histoire de l'architecture}

$37 \mid 2019$

L'architecture mise à l'examen

\title{
Les concours à l'académie de Saint Ferdinand : méthodes et périodes d'une nouvelle culture architecturale en Espagne (1753-1808)
}

Competitions at the Academy of Saint Ferdinand. Methods and periods of a new architectural culture in Spain 1753-1808)

Die Concours an der Real Academia de Bellas Artes de San Fernando. Methoden und Konjunkturen einer neuen Architekturkultur in Spanien (1753-1808)

\section{Adrián Almoguera}

\section{OpenEdition}

\section{Journals}

Édition électronique

URL : http://journals.openedition.org//ha/1367

DOI : $10.4000 /$ /ha. 1367

ISSN : 1960-5994

Éditeur

Association Livraisons d'histoire de l'architecture - LHA

Édition imprimée

Date de publication : 15 juin 2019

Pagination : 23-34

ISSN : $1627-4970$

\section{Référence électronique}

Adrián Almoguera, « Les concours à l'académie de Saint Ferdinand : méthodes et périodes d'une nouvelle culture architecturale en Espagne (1753-1808) », Livraisons de l'histoire de l'architecture [En ligne], 37 | 2019, mis en ligne le 02 janvier 2021, consulté le 25 janvier 2021. URL : http:// journals.openedition.org//ha/1367 ; DOI : https://doi.org/10.4000//ha.1367 


\section{LES CONCOURS À L'ACADÉMIE DE SAINT-FERDINAND : MÉTHODES ET PÉRIODES D'UNE NOUVELLE CULTURE ARCHITECTURALE EN ESPAGNE (1753-1808)}

Dès son ouverture officielle en 1752, l'Académie royale des Beaux-Arts de Saint-Ferdinand de Madrid a eu pour but d'être un lieu de formation pour la régénération des arts nationaux, considérés par les élites espagnoles comme beaucoup trop en retard et contrôlés par des spécialistes étrangers ${ }^{1}$. En effet, l'académie de Madrid était devenue un instrument de pouvoir s'exerçant sur la production artistique du pays et sa réforme symbolisait le bon gouvernement d'une monarchie éclairée et bienfaisante. Or, si les statuts de cette institution préconisaient une aequa potestas artistique à la romaine, c'est-à-dire, une supposée égalité entre les trois arts, c'est la réforme de l'architecture qui concentre les plus gros efforts. La construction du système académique madrilène s'est ainsi basée sur une combinaison sélective des modèles académiques parisien et romain. L'histoire comparée de ces trois institutions nous montre comment le modèle de concours académiques franco-italien a été important pour le développement d'une pédagogie académique en Espagne ${ }^{2}$. Dans ce contexte, les concours d'architecture organisés par l'académie de Madrid sont devenus l'un des moteurs du renouveau de l'art de bâtir dans ce pays. On regrettera que leur étude n'ait pas fait l'objet de recherches suffisamment poussées, surtout dans leur rapport avec le contexte international ainsi qu'avec les débats d'une réalité constructive espagnole en pleine effervescence à la fin des Lumières.

\section{Inventer un système : l'académie de Madrid entre Rome et Paris}

Les concours madrilènes étaient organisés en trois classes de difficulté croissante, de la troisième et plus simple, à la première et plus prestigieuse. Mis en place pour la première fois en 1753 avec pour but d'être annuels, ils s'interrompent en 1754, reprennent entre 1755 et 1757 , puis deviennent triennaux jusqu'en 1808 avec une interruption entre 1772 et $1778^{3}$. Les concours madrilènes ont été très peu inter-

1. Claude Bédat, L'Académie des Beaux-Arts de Madrid, Toulouse, Toulouse-Le Mirail, 1973, 478 p.

2. Voir notamment Basile Baudez, Architecture et tradition académique, Rennes, PUR, 2012, 390 p., ainsi que les autres publications de l'auteur en matière de culture architecturale européenne comparée.

3. Interrompus à nouveau en 1808, ils sont convoqués une dernière fois en 1831. Voir la liste complète dans Delfín Rodríguez, Hacia una nueva idea de la Arquitectura : Premios generales de Arquitectura de la Real Academia de Bellas artes de San Fernando 1753-1831, Madrid, RABASF, 1992, p. 181-187. 
nationaux car en soixante ans, seulement deux étrangers, Virgilio Verda et François Claude Billard de Bélisard, ont remporté les prix de 1756 et 1763 respectivement. Il est clair que dans l'Europe de l'époque les concours italiens étaient les plus attrayants, notamment ceux de Rome (Clementino et Balestra), mais aussi ceux organisés par la jeune Académie de Parme, créée en 1757, dont les concours avaient la renommée d'être plus avant-gardistes et plus égalitaires que ceux de la toute puissante Accademia de San Luca ${ }^{4}$. À Madrid, les épreuves s'ouvraient à l'ensemble de candidats souhaitant concourir puisque le but principal était de répandre les «Lumières académiques " sur tout le territoire national. Elles étaient divisées en deux temps : d'abord une première prueba de pensado, équivalente au elaborato finale romain, de plusieurs mois de durée, faite hors l'institution (et donc sans contrôle des conditions de son élaboration), et dont les projets devaient être rendus à l'académie suivant une stricte liste de règles. S'ensuivait une prueba de repente, équivalente au squizzo à Rome, de quelques heures de durée, réalisée à l'intérieur de l'académie sous la surveillance des enseignants permettant ainsi de confirmer l'originalité et la paternité des grands projets rendus 5 .

Du point de vue de leur utilité professionnelle, ces concours se rapprochaient plutôt du modèle parisien. Contrairement à Rome, où ces rencontres académiques servaient surtout à couronner une carrière d'une récompense prestigieuse très recherchée par les architectes internationaux, les concours madrilènes devaient certifier et célébrer l'acquisition d'un ensemble de connaissances architecturales diffusées par l'académie avec une claire finalité pratique ${ }^{6}$. Cette culture architecturale devait servir à répandre partout en Espagne les dictats de l'institution grâce à ces architectes qui, une fois vainqueurs, partaient travailler dans les provinces sous la surveillance académique, en tant que professionnels d'un système artistique d'État.

La composition et le style des projets rendus dans ces concours de l'académie de Saint-Ferdinand révèlent des problématiques très intéressantes quant à la circulation de modèles architecturaux dans l'Europe de cette époque. En ce qui concerne les aspects artistiques des dessins, comme l'usage de la couleur et des effets pittoresques, la tendance à la monochromie des rendus est assez stable. Ceci peut s'expliquer non seulement par la méfiance des académiciens envers la "contamination » des effets artistiques ou pittoresques qui abondaient dans l'architecture espagnole "préacadémique ", mais également par la manière dont les jeunes élèves apprenaient à dessiner à Saint-Ferdinand, souvent très axée sur l'étude et la copie répétitive des

4. L'ensemble de projets présentés aux concours de Rome se trouve dans Paolo Marconi, Angela Cipriani, Enrico Valeriani, I disegni di architettura dell'Archivio storico dell'Accademia di San Luca, Rome, De Luca, 1974, 2 vol., 887 p. À propos de Parme, voir Carlo Mambriani, "Un'alternativa alle corone di San Luca : i concorsi dell'Accademia di Parma tra 1780 e 1800 ", Susanna Pasquali (dir.), Contro il Barocco, Roma, Campisano, 2007, p. 109-117.

5. Le déroulement des épreuves est résumé dans Silvia Arbaiza Soler, Carmen Heras Casas, "Inventario de los dibujos de arquitectura de los siglos XVIII y XIX en la RABASF (I) », Academia, n 110-111, 2010, p. 89-119.

6. Damie Stillman, "British Architects and Italian Architectural Competitions, 1758-1780 ", Journal of the Society of Architectural Historians, vol. 32, $\mathrm{n}^{\circ}$ 1, 1973, p. 43-66. 
planches monochromes des traités et des recueils d'architecture canoniques ${ }^{7}$. Néanmoins, bien que les projets de Saint-Ferdinand n'aient jamais atteint le côté spectaculaire des projets parisiens de la fin du XVIII ${ }^{\mathrm{e}}$ siècle, on constate une certaine " réception " de ces effets dessinés dans les rendus de plusieurs concours madrilènes, comme celui de 1805, qui demandait «Trois lazarets pour Madrid» pour la première classe, et «Un gymnase pour la jeunesse d'Espagne à ériger aux alentours de la capitale " pour la seconde.

Quant à la composition des projets, l'étude de ce fonds de dessins d'architecture a révélé une véritable richesse dans l'assimilation et la réinterprétation des modèles internationaux, surtout italiens et français, diffusés par les ouvrages imprimés, mais aussi très présents dans les envois de Rome que les pensionnaires de l'académie amenaient à Madrid enrichis des nouveautés acquises dans le milieu international romain. Pour les concours, les élèves de Saint-Ferdinand ont beaucoup regardé les recueils de gravures d'architecture qui circulaient en Europe dans cette période, de l'Histoire de l'architecture de Fischer Von Erlach (1721), à la Collection des Prix de l'Académie royale d'architecture, publiés en France à partir de 1787 (ill. 1-2) ${ }^{8}$. L'évolution évidente et rapide dans les méthodes de composition des rendus des concours académiques madrilènes montre que s'est imposée une progressive "abstraction géométrique " dans la pensée architecturale développée au sein de l'académie. Cette abstraction, encore plus évidente dans les concours parisiens et romains de la fin du siècle, impose un principe de symétrie qui articule des bâtiments de plus en plus complexes, devenus de véritables machines architecturales dont la multiplicité des espaces et des fonctions disposés sur le plan reflète les besoins du nouvel ordre social qui les commande ${ }^{9}$. Fière des progrès acquis dans cette matière, à partir de l'été de 1793 l'académie madrilène commence à exposer publiquement les meilleurs projets vainqueurs des concours dans le but de rendre publics les progrès des arts nationaux acquis sous l'égide de l'académie et sous la haute protection de la couronne ${ }^{10}$

L'un des buts principaux de ces concours était de définir un classicisme espagnol afin d'impulser une nouvelle architecture nationale. Ce classicisme émerge à la fin

7. Nous avons abordé ce sujet dans Adrián Almoguera, "Entre las luces y el antiguo : le dessin d'architecture à l'Académie des Beaux-Arts de Saint-Ferdinand, 1752-1815 ", Livraisons d'histoire de l'architecture, $\mathrm{n}^{\circ} 29$, décembre 2016, p. 33-47.

8. À propos de cette publication, voir Helen Rosenau, "The Engravings of the Grands Prix of the French Academy of Architecture ", Architectural history, n 3, 1960, p. 17-180 et plus récemment Marie-Laure Crosnier-Leconte, "Dessins d'école, bibliothèques d'ateliers : une affaire de copies ", dans Jean-Philippe Garric (dir.), Bibliothèques d'atelier. Édition et enseignement de l'architecture, Paris, 1785-187, INHA ( Les catalogues d'exposition de l'INHA»), 2011, [En ligne], mis en ligne le 28 avril 2011, consulté le 29 janvier 2019. URL : http://journals.openedition.org/inha/3185.

9. Sur les nouveautés dans la composition de ces projets dans l'axe Rome-Paris, et au-delà des publications classiques de Jean-Marie Pérouse de Montclos, voir Gian Paolo Consoli, "Verso una nuova architettura : Académie Royale d'architecture e Accademia di San Luca 1750-1800 ", Roma-Parigi, accademie a confronto, Roma, ANSL, 2017, p. 81-104.

10. Archives de l'Académie de Saint-Ferdinand, désormais ASF, 1-55-2, Euvres d'art des professeurs des Beaux-Arts exposées au public pendant les mois d'été entre 1793 et 1808. 


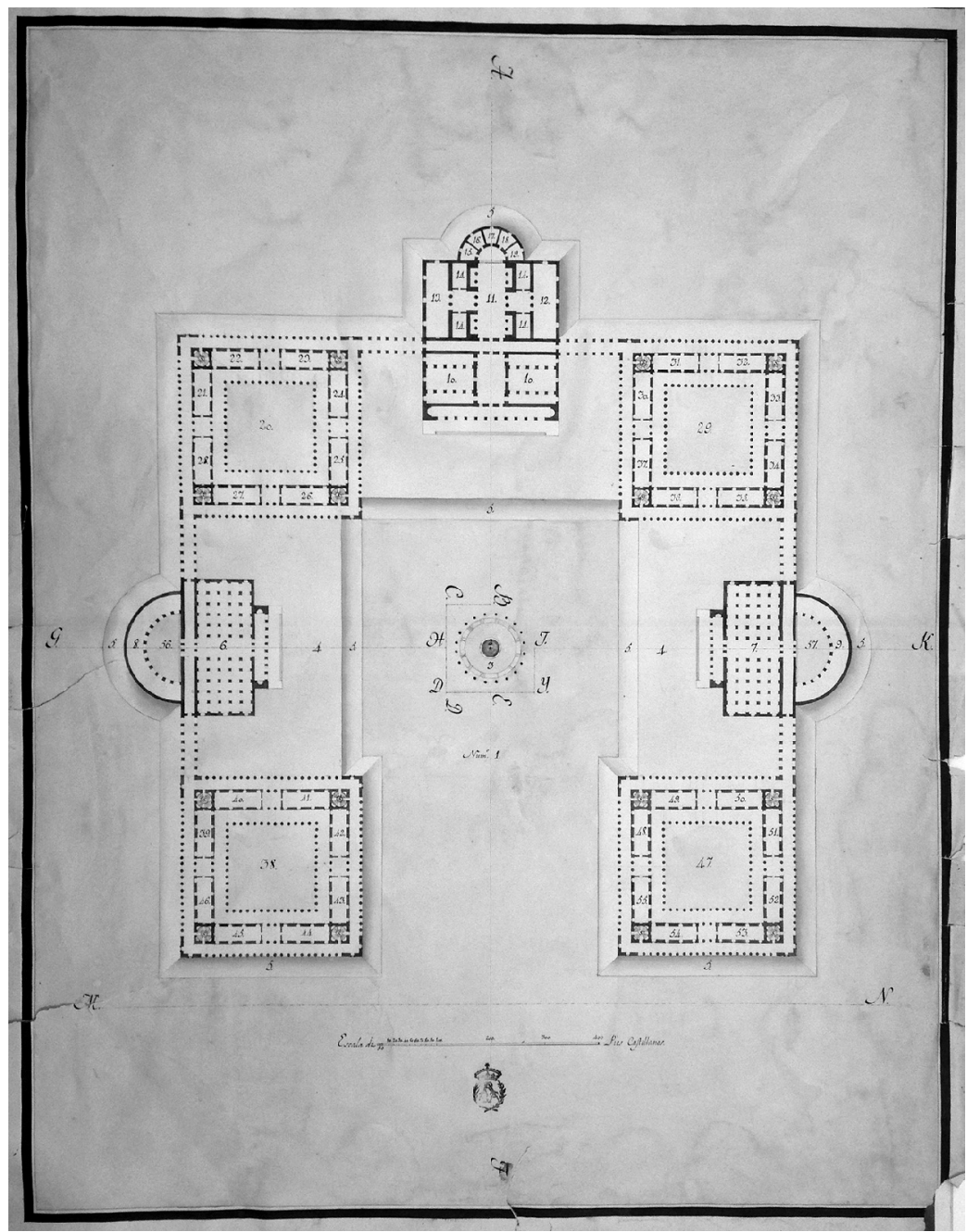

Ill. 1 : Cayetano Gallitia, Projet de marché public, Premier prix de deuxième classe du concours général de 1799, $107 \times 75 \mathrm{~cm}$, Madrid, RABASF, (C) Madrid, Real Academia de Bellas Artes de San Fernando, A-2289.

des Lumières, dans l'architecture dessinée pour ces concours et est le résultat de la combinaison théorique et pratique de plusieurs modèles.

La réflexion se développe parallèlement dans les débats et les écrits théoriques de l'académie ${ }^{11}$. Le style recherché est d'abord nourri des références au classicisme

11. La question des classicismes dans l'académie est présente dans l'ensemble d'articles publiés par Carlos Sambricio, La arquitectura española de la Ilustración, Madrid, COAM, 1986, 491 p., et dans l'étude d'introduction de Delfín Rodríguez, Hacia una nueva idea de la Arquitectura..., op. cit., p. 15-31. 


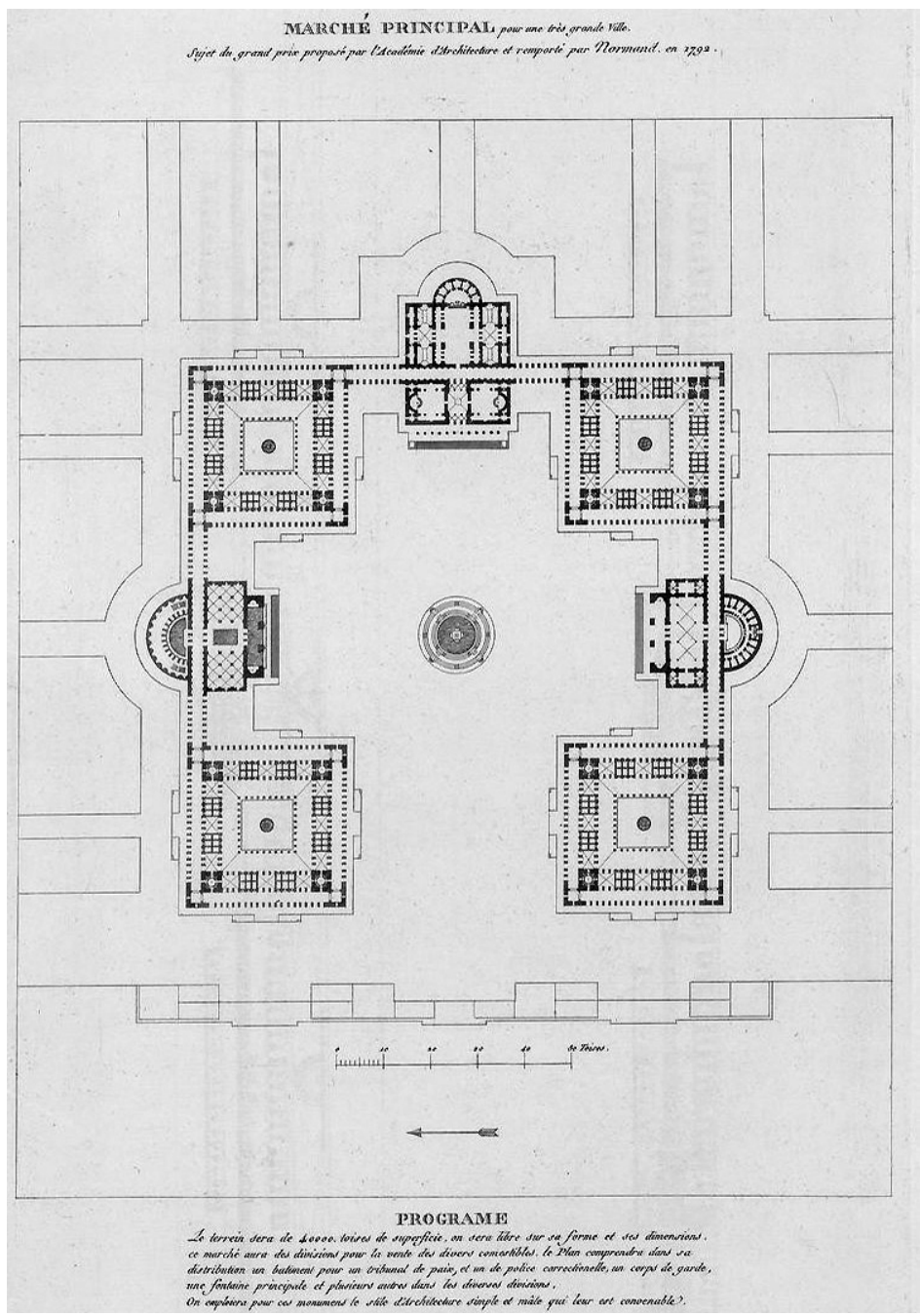

Ill. 2 : Marché Principal pour une très grande ville, sujet du Grand Prix proposé par l'Académie d'Architecture et remporté par Normand en 1792, Allais, Détournelle et Vaudoyer, Grands Prix d'architecture..., Paris, 1802, (C) DR.

italien et au système des ordres architecturaux, de Vitruve à Vignole et Palladio, dont les traductions et les rééditions occupent une place importante dans les publications de l'académie ${ }^{12}$. On y retrouve aussi l'Antiquité revisitée et réinterprétée,

12. Sur les traductions au sein de l'académie voir Delfín Rodríguez, "José Ortiz y Sanz, "atención y pulso" de un traductor ", prologue à l'édition de Vitruve, Diez Libros de Arquitectura, traducidos al castellano por José Ortiz y Sanz, Madrid, Akal, 2001, p. 7-33. 


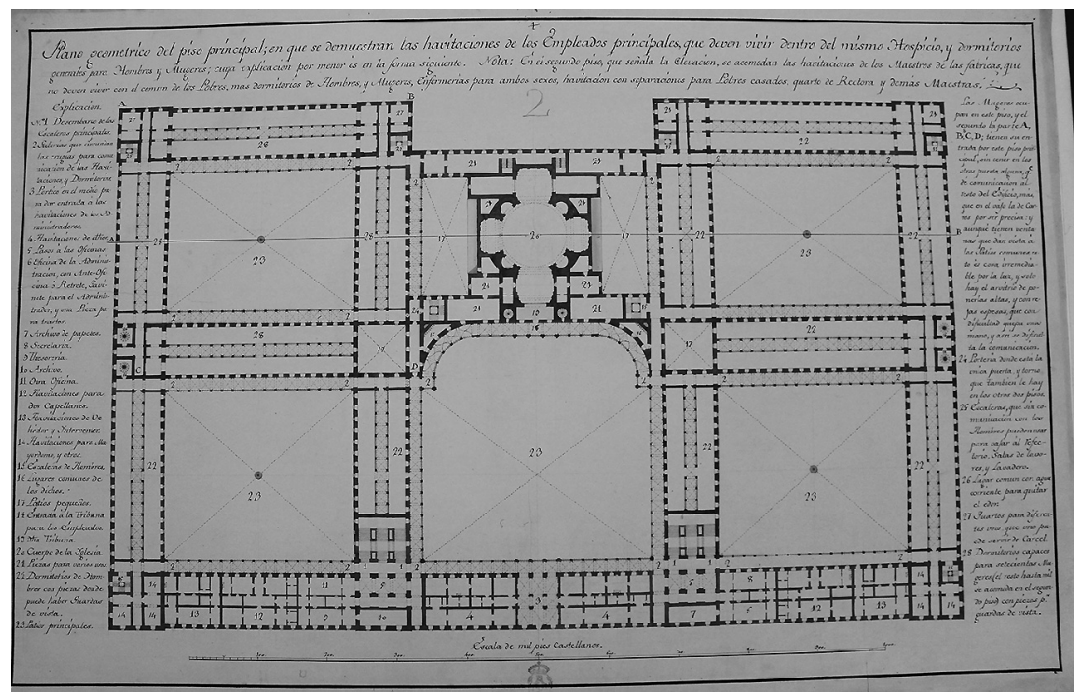

Ill. 3 : Guillermo Casanova, Projet pour un grand hôpital (plan général), Premier prix de première classe du concours général de 1778, $61 \times 94 \mathrm{~cm}$, Madrid, RABASF, C Madrid, Real Academia de Bellas Artes de San Fernando, A-2666.

surtout par les architectes pensionnaires à Rome qui ramènent à Madrid des dessins et des moulages des monuments anciens. Ils sont riches d'un bagage théorique cosmopolite, diffusent des publications, exposent des envois, et contribuent au bon déroulement des concours académiques en occupant des fonctions d'enseignement dans l'institution ${ }^{13}$. À ces deux modèles, qui ont marqué les étapes de l'évolution architecturale des académies européennes de Paris à Saint-Pétersbourg, le cas espagnol rajoute une troisième référence fondamentale qui fait sa différence : le classicisme épuré et unique de Juan de Herrera, représenté par le modèle incontournable de l'Escurial (ill. 3) ${ }^{14}$. C'est effectivement ce mélange entre le classicisme international de la Renaissance, le classicisme intemporel de l'Antiquité et le classicisme autochtone représenté par les leçons de Juan d'Herrera à l'Escurial qui a fait la renommée des plus grands chefs-d'œuvre de l'architecture néoclassique espagnole de la période. Parmi eux, s'imposent sans doute les projets madrilènes de Juan de Villanueva, vainqueur de la seconde classe du concours général de 1756, pensionnaire à Rome entre 1759 et 1764 puis professeur à l'académie, à propos desquels Francesco Milizia remarquait " la pureté du goût fondé sur la simplicité grecque », et qui mettait toujours en évidence son titre de premier architecte de l'ordre de Saint Jérôme à l'Escurial, où il bâtit ses premiers édifices d'importance à partir de $1768^{15}$.

13. Sur les pensionnaires à Rome, voir Pedro Moleón, Arquitectos españoles en la Roma del Grand Tour, Madrid, Abada, 2004, 416 p.

14. L'importance de l'Escurial est brillamment soulignée par Delfín Rodríguez, "La sombra de un edificio : El Escorial en la cultura arquitectónica española durante la época de los primeros Borbones (1700-1770) ", Quintana, n² 2, 2003, p. 57-94.

15. Francesco Milizia, Memorie degli architetti antichi e moderni, t. II, Bassano, 1785, p. 312. 


\section{Définir et promouvoir une architecture publique : l'académie à la rencontre de la modernité}

Au-delà des aspects artistiques et stylistiques, les programmes des concours montrent le rôle qu'ils jouent dans l'élaboration d'une nouvelle architecture publique pensée pour la société issue du progressif effondrement de l'Ancien Régime. L'étude de l'évolution des programmes à Madrid reflète effectivement le lien, silencieux mais effectif, entre l'architecture publique académique et les nouveautés sociales et culturelles du tournant du XIX ${ }^{\mathrm{e}}$ siècle. On assiste à la naissance d'une considérable réflexion théorique et pratique qui se traduit dans le renouveau total des typologies architecturales étudiées au sein des académies européennes de la période, y compris celle de Madrid. Dans ce contexte, entre 1750 et la suppression révolutionnaire de 1793, l'Académie royale de Paris produit un total de 320 programmes thématiques pour la composition architecturale, alors que Rome en proposait 26 jusqu'au célèbre et polémique Concorso Clementino de 1795, remporté ex-aequo pour la première fois dans son histoire par l'espagnol Jorge Durán ${ }^{16}$.

À Madrid, entre 1754 et 1808 , cent quatre-vingts programmes sont proposés, la deuxième et surtout la troisième classe ayant tendance à reproduire les programmes des concours qui peuvent être regroupés par catégories. La première classe est presque toujours réservée aux programmes les plus prestigieux, en lien avec le pouvoir ou les besoins sociaux de la période. La deuxième classe propose d'abord l'élaboration d'objets architecturaux plus concis dépendants d'un programme majeur tels qu'une chapelle, un escalier, une cour ou une façade. Néanmoins, cette deuxième classe acquiert à partir des années 1760 une plus grande importance lorsqu'elle commence à proposer des programmes plus élaborés comme des arènes (1763), un marché public (1769), en théâtre en pierre et brique (1772), un cimetière (1784) ou un gymnase public (1805). La troisième classe évolue moins, et ses programmes varient entre la copie de bâtiments et de monuments madrilènes représentatifs, et surtout l'étude et la copie de certains éléments architecturaux comme les architraves ou les frontispices, extraits des principaux traités en la matière. Il faut signaler que, malgré l'intention pédagogique de ces concours à l'échelle nationale, la capitale ne s'est jamais posé la question de leur diffusion comme ce fut le cas de la célèbre Collection des prix de l'académie parisienne qui a inspiré les Prix remportés par les élèves de l'académie impériale des Beaux-Arts de Saint-Petersburg publiés en 1805, ainsi que d'autres initiatives similaires comme la Raccolta di IX progetti architettonici proposés par l'Accademia della Pace, et publiés à Rome dans un recueil gravé en $1795^{17}$. À Madrid, les efforts pour la conservation et la diffusion des projets présentés aux concours académiques se sont limités à la constitution d'une collection académique

16. Basile Baudez, Architecture et tradition..., op. cit., p. 231. Sur le concours de 1795, Adrián Almoguera, "Il Concorso Clementino di 1795 ", Roma-Parigi, accademie..., op. cit., p. 210-213.

17. Sur ce dernier, voir Susanna Pasquali, "Contributo alla conoscenza della cultura architettonica a Roma alla fine del '700 : Felice Giani e l'Accademia della pace ", Quaderdi del Dipartimento de patrimonio architettonico e urbanistico, $\mathrm{n}^{\circ} 1,1992$, p. 76-88. 
regroupant l'ensemble des rendus, à laquelle on a rajouté les dons, et les envois de Rome. L'académie devenait ainsi la principale gardienne du patrimoine dessiné. Celui-ci témoignait de la transformation de la pensée architecturale espagnole de la fin des Lumières, marquée par l'essor d'une nouvelle architecture civile désormais débattue et modelée dans les concours académiques. Dans cette perspective, l'étude des sujets proposés par les membres de l'académie se révèle fondamentale pour comprendre la manière dont l'académie de Madrid a pu servir de «laboratoire d'expérimentation " architecturale, bien que le sens de cette expression soit limité dans le cas de ces manifestations très encadrées par des règlements stricts et dont le but principal était de plaire à un jury ${ }^{18}$.

Les programmes sur l'architecture religieuse méritent une mention plus particulière dans le contexte de la réforme nationale des temples chrétiens espagnols entreprise par Charles III dès $1777^{19}$. Entre 1752 et 1808 , onze programmes concernent directement un bâtiment ou objet architectural religieux, dont neuf demandent qu'il soit de la propre invention du candidat ${ }^{20}$. La cathédrale n'apparaît dans la première classe qu'en 1808, certainement à cause du contexte d'exhortation nationaliste contre l'imminente invasion française. Nous trouvons surtout des programmes concernant les tabernacles et les autels, objets très importants dans la réforme de l'architecture religieuse entreprise par Charles III qui montrent une évolution réelle des formes architecturales depuis les modèles baroques jusqu'aux propositions plus sobres basées sur un classicisme très épuré en strict lien avec les nouvelles doctrines du christianisme éclairé. Néanmoins, la plupart de sujets d'architecture sacrée apparaissent dans les pruebas de repente, et leur impact est surtout important pour l'architecture des provinces puisqu'à Madrid le dernier grand débat sur un bâtiment religieux s'éteint avec l'achèvement de la basilique de Saint-Françoisle-Grand en 1784.

Cette évolution est symptomatique d'une autre, plus générale, qui concerne les principaux programmes des deux premières classes. Ainsi, dans les premiers temps de l'académie les programmes de première classe célèbrent d'abord les pouvoirs de la monarchie absolue représentée par l'église dédiée au roi (1753), le palais princier (1754) où le palais de l'archevêché (1763). Néanmoins, bien que ces projets ne disparaissent pas complètement, une nouvelle architecture civile et publique se profile dès la fin des années 1760, pour se consolider et triompher définitivement au tournant du XIX ${ }^{\mathrm{e}}$ siècle sous les auspices des théories de Francesco Milizia et la direction académique des anciens pensionnaires à Rome. Ainsi, la bibliothèque publique (1787), le collège universitaire (1790), la douane maritime (1793), les cimetières

18. La question des concours "laboratoires" est abordée depuis de nombreux points de vue par Basile Baudez, Architecture et tradition académique..., op. cit., p. 220-306.

19. Sur la question de l'architecture religieuse espagnole voir A. García de Ceballos, «La reforma de la arquitectura religiosa en el reinado de Carlos III. El neoclasicismo español y las ideas jansenistas ", Fragmentos, $\mathrm{n}^{\circ} 12-13-14,1988$, p. 115-127.

20. À cela il faut rajouter certains programmes "mixte " comme les collèges universitaires, ou les hôpitaux, qui incluent généralement des églises et des chapelles dans leur ensemble. 
(1799) et les hôpitaux (1805) pour la première classe, ainsi que les marchés (1769, 1799), la bourse de commerce (1796), les maisons de bains (1781, 1793), et le gymnase public pour la jeunesse (1805) pour la deuxième, imposent progressivement un nouveau langage de modernité architecturale imprégné des nouveautés du contexte international ${ }^{21}$. Les propositions faites par les candidats de la fin du siècle transforment le projet architectural académique en un éloge des volumes épurés érigés sur des vastes plans composés d'après un nouvel ordre de géométrie et de monumentalité. Réflexion architecturale et culturelle sont menées de pair, et chaque typologie d'édifice étudié dans les concours reflète davantage les préoccupations liées à sa fonctionnalité et à son rôle dans l'ensemble de la machinerie sociale de l'État en construction. Un exemple clair est celui du projet d'académie de Beaux-Arts, proposée comme sujet de première classe en 1756, quand l'académie fait ses premiers pas et définit ses statuts et sa politique pédagogique, et de nouveau en 1796, époque de la fin abrupte de l'académie parisienne qui remet en question ce système en Europe alors qu'à Madrid l'académie traverse une crise pédagogique et méthodologique importante ${ }^{22}$. Ces deux sujets successifs nous invitent à réfléchir sur la manière dont l'académie a théorisé et expérimenté sa propre forme architecturale, et par extension, sur l'image qu'elle devait offrir en tant qu'institution d'État essentielle pour la réforme de la culture nationale. Ainsi, le projet d'académie de Virgilio Verda, lauréat du concours en 1756, est étroitement lié à l'architecture du chantier du nouveau palais royal madrilène. Il concentre l'essentiel de la réflexion architecturale sur ces formes princières qui lient l'académie au prestige de la monarchie qui l'a créée (ill. 4). Quarante ans plus tard, Agustín de Humarán remporte un premier prix avec une académie qui adopte la forme d'un immense bâtiment carré autour d'une grande place centrale, avec douze portiques monumentaux, et une complexe distribution où se succèdent les espaces parfaitement géométriques qui mélangent les rotondes et les salles basilicales, les espaces d'exposition artistique et les salles aux fonctions multiples. Ce projet vainqueur donnait ainsi l'image d'une académie universaliste et moderne, dont l'architecture à l'antique représentait un projet régénérateur pour les arts de la nation (ill. 5).

Au regard de ces expérimentations, peut-on affirmer que les concours académiques de Madrid ont créé des "types" architecturaux ? Pour répondre à cette question, il faudrait approfondir davantage les différences entre architecture dessinée et architecture construite. Sur le papier, à la fin des Lumières, on trouve effectivement à Madrid des "types " qui se répètent pour certains édifices comme les hôpitaux, les cimetières ou les théâtres. Parfois, surtout dans la première moitié du XIX siècle, c'est un bâtiment construit qui crée un «type " académique, comme c'est le cas du

21. Cette "définition d'une architecture publique" est soulignée par Jean-Marie Pérouse de Montclos, Les Prix de Rome : concours de l'Académie royale d'architecture au XVIII siècle, Paris, ENSBA, 1984, p. 15-20, ainsi que par Basile Baudez, Architecture et tradition..., op. cit., p. 131-251.

22. Les difficultés de l'académie dans la décennie de 1790 sont étudiées par Enrique García Melero (dir.), Renovación, crisis, continuismo : la Real Academia de San Fernando en 1792, Madrid, RABASF, 1992, $158 \mathrm{p}$. 


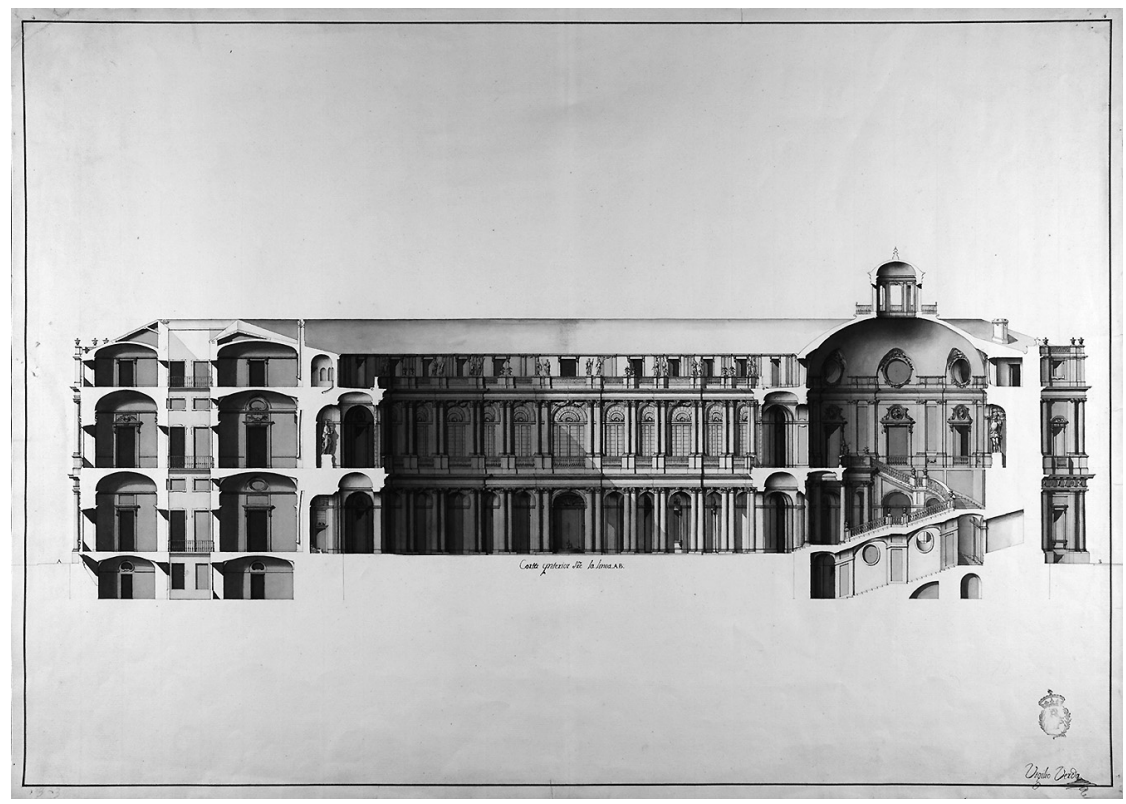

Ill. 4 : Virgilio Verda, Projet d'académie pour les trois nobles arts, Premier prix de première classe du concours général de 1756 (coupe longitudinale), $53 \times 74 \mathrm{~cm}$, Madrid, RABASF, (C) Madrid, Real Academia de Bellas Artes de San Fernando, A-0145.

Cabinet royal de sciences conçu dès 1785 par Juan de Villanueva qui est repris de nombreuses fois au sein des examens académiques (ill. 6). Néanmoins, la transposition entre cette architecture publique dessinée et sa version construite reste, à quelques exceptions près, bien moins évidente que pour certains programmes français, comme les palais de justice, dont les caractéristiques architecturales demeurent tout au long du XIX ${ }^{\mathrm{e}}$ siècle, assez fidèles au modèle créé par l'académie royale de Paris $^{23}$. Remarquons que la matérialisation de ces "types " académiques en France a été en grande partie possible grâce à l'intervention du Conseil des bâtiments civils. Ce n'est pas le cas en Espagne car même si l'académie possédait depuis 1786 un organisme très similaire, la Comisión de arquitectura, elle n'a pas réussi à développer le moindre "type" précis pour une typologie concrète susceptible de consacrer définitivement, sur le terrain, les expérimentations architecturales des concours académiques. Les transpositions entre concours et réalité constructive sont restées très limitées, et ont surtout concerné la reprise de certains éléments novateurs comme des amphithéâtres anatomiques sur le modèle de l'école de chirurgie de Jacques Gondoin, repris plusieurs fois dans les concours madrilènes, et que l'académicien Ignacio Haan reproduit, par exemple, dans l'hôpital général de Madrid dans les années 1790 . Ce qui ne veut pas dire que les concours académiques n'ont pas eu 


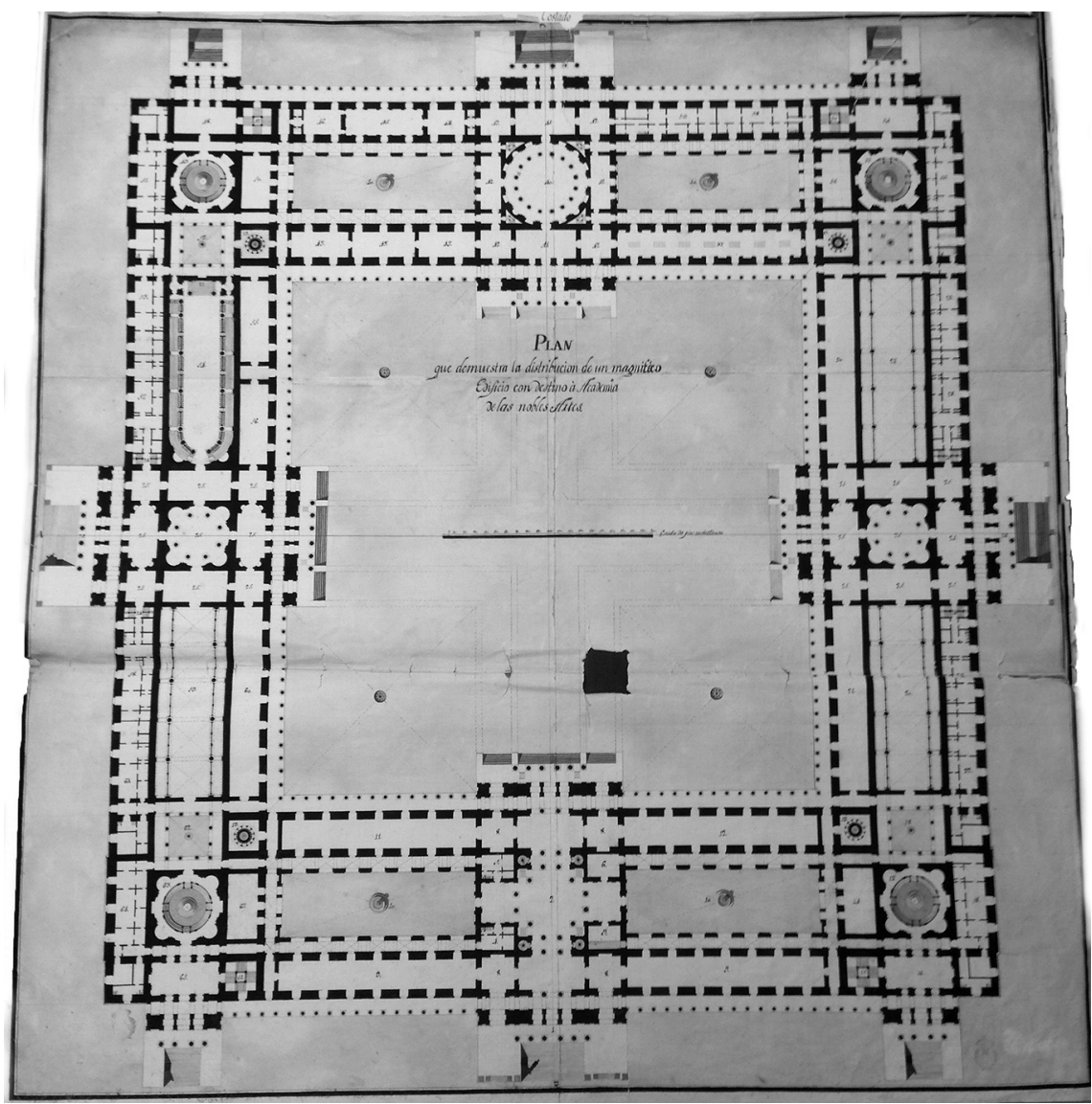

Ill. 5 : Agustín de Humarán, Projet d'académie pour les trois nobles arts, Premier prix de première classe du concours général de 1796 (plan), $95 \times 96 \mathrm{~cm}$, Madrid, RABASF, (C) Madrid, Real Academia de Bellas Artes de San Fernando, A-0214.

une certaine influence sur la réalité constructive espagnole. En effet, les dernières recherches faites dans cette perspective à partir du fonds de dessins et des archives de la Comisión de arquitectura, chargée de conduire tous les débats sur la construction de typologies scientifiques, hygiénistes ou culturelles pour la ville, prouvent que le lien entre les programmes des concours, par exemple ceux de 1769, 1799 ou 1805, et l'actualité architecturale madrilène a été très étroit dans un certain nombre d'occasions dont l'étude dépasse l'étendue du présent article.

Ainsi, entre projets rêvés et initiatives concrètes, entre propositions purement académiques et idées visant à créer une véritable nouvelle manière de bâtir, les concours de l'académie de Saint-Ferdinand sont l'un des exemples majeurs du renouveau de l'art de bâtir en Espagne à la fin des Lumières. Leurs caractéristiques stylistiques et techniques font preuve d'une culture artistique de plus en plus parta- 


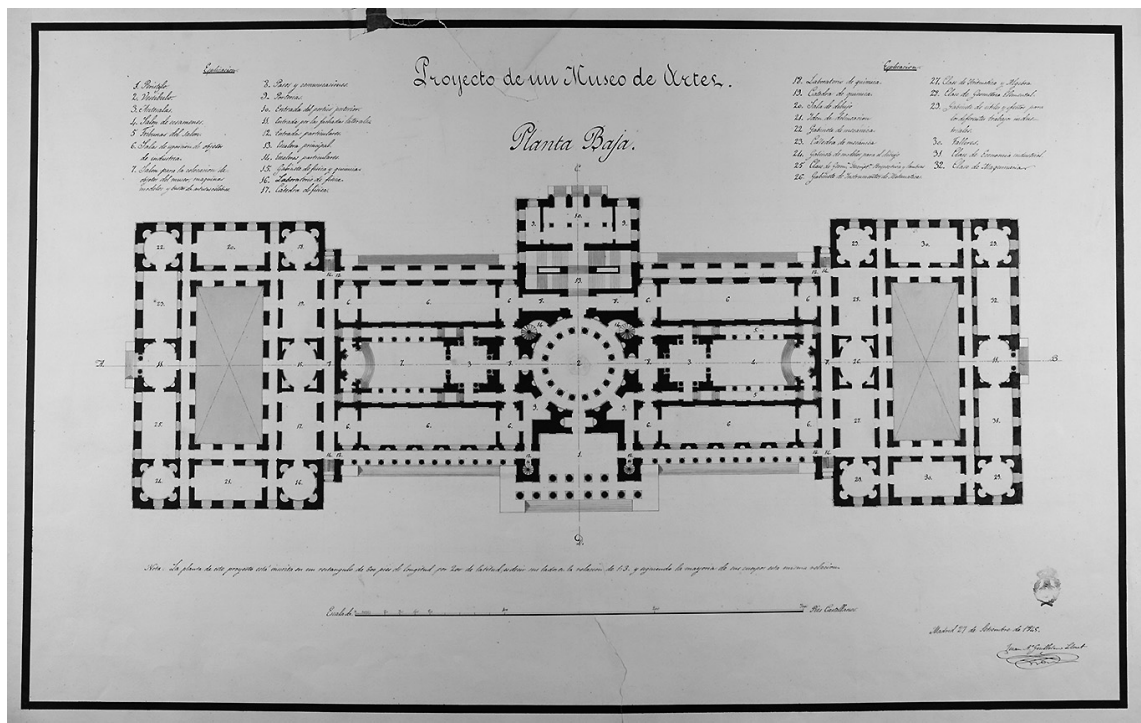

Ill. 6 : Juan Antonio Lloret, Projet pour un musée d'art (plan principal), 1845, $62 \times 97 \mathrm{~cm}$, Madrid, RABASF, (C) Madrid, Real Academia de Bellas Artes de San Fernando, A-0053.

gée en Europe, tendant à une homogénéisation internationale encore plus évidente dans la composition et la forme de ces projets. Ils ont été un espace d'expérimentation et d'application de formules visant à la définition d'un nouveau classicisme architectural, international et autochtone à la fois. Un classicisme symboliquement et artistiquement orienté vers la création d'une architecture publique et civile qui a nourri le débat académique et social autour de 1800, et qui s'incarne, décliné de manières infinies, dans de nombreuses constructions de la capitale et des provinces espagnoles de la première moitié du XIX ${ }^{\mathrm{e}}$ siècle.

Adrián Almoguera

Doctorant, Université Paris IV-Sorbonne/Centre André Chastel Lecteur en art, histoire et civilisation espagnole et hispano-américaine Université Paris-Sorbonne 